\title{
A Novel Process-Compatible Fluorination Technique With Electrical Characteristic Improvements of Poly-Si TFTs
}

\author{
Shen-De Wang, Student Member, IEEE, Wei-Hsiang Lo, Tzu-Yun Chang, and Tan-Fu Lei
}

\begin{abstract}
A process-compatible fluorine passivation technique of poly-Si thin-film transistors (TFTs) was demonstrated by employing a novel $\mathrm{CF}_{4}$ plasma treatment. Introducing fluorine atoms into poly-Si films can effectively passivate the trap states near the $\mathrm{SiO}_{2} /$ poly-Si interface. With fluorine incorporation, the electrical characteristics of poly-Si TFTs can be significantly improved including a steeper subthreshold slope, smaller threshold voltage, lower leakage current, higher field-effect mobility, and better On/Off current ratio. Furthermore, the $\mathrm{CF}_{4}$ plasma treatment also improves the reliability of poly-Si TFTs with respect to hot-carrier stress, which is due to the formation of strong $\mathrm{Si}-\mathrm{F}$ bonds.
\end{abstract}

Index Terms- $\mathrm{CF}_{4}$ plasma, fluorine, fluorine passivation, poly-Si, process-compatible, reliability, TFTs.

\section{INTRODUCTION}

$\mathbf{P}$ OLYSILICON THIN-FILM transistors (poly-Si TFTs) have attracted much attention due to the possibility of realizing the integration of driving circuits and pixel elements on one glass substrate, and the potential to accomplish the system-on-panel (SOP) [1]. High-performance poly-Si TFTs are required for this goal. A hydrogenation process has been utilized to reduce the trap states of poly-Si films to improve the device performance [2]. However, hydrogenated poly-Si TFTs suffer from a serious instability issue due to weak $\mathrm{Si}-\mathrm{H}$ bonds, which will break easily under an electrical stress [3]. Using fluorine to passivate the trap states of poly-Si TFTs has been reported recently [4]-[6]. Strong Si-F bonds, more stable than $\mathrm{Si}-\mathrm{H}$ bonds, can maintain the device performance under long-term electrical stress. Conventional fluorine ion implantation might be not suitable for large-sized glass substrate applications. Moreover, a subsequent high temperature annealing is required to activate the fluorine atoms and recover the defects created by ion implantation. However, high-temperature process is not compatible with current production due to the low-melting point of low-cost glass substrate. Kim et al. demonstrated the use of fluorinated oxide $\left(\mathrm{SiO}_{x} \mathrm{~F}_{y}\right)$ to serve as a diffusion source [7], [8]. However, an extra film deposition and etching are required. To date, although hydrogen-based plasma treatments have been widely investigated in poly-Si TFTs, there

Manuscript received February 22, 2005; revised March 18, 2005. This work was supported by the National Science Council of Taiwan, R.O.C., under Contracts NSC 93-2215-E-009-003 and NSC 93-2215-E-009-036. The review of this letter was arranged by Editor J. Sin.

The authors are with the Department of Electronics Engineering and Institute of Electronics, National Chiao-Tung University, Hsinchu 300, Taiwan, R. O. C.

Digital Object Identifier 10.1109/LED.2005.848095 is still a lack of an investigation of the fluorine-based plasma treatment.

In this letter, a novel fluorination technique is proposed by employing a low-temperature $\mathrm{CF}_{4}$ plasma treatment. This technique provides a simple, effective and process-compatible method to introduce fluorine atoms into poly-Si films and high-performance and high-reliability poly-Si TFTs were fabricated.

\section{EXPERIMENTAL}

The schematic diagram of fabrication processes is illustrated in Fig. 1. First, a 100-nm-thick amorphous silicon layer was deposited on a thermally oxidized $\mathrm{Si}$ wafer by dissociation of $\mathrm{SiH}_{4}$ gas in a low-pressure chemical vapor deposition (LPCVD) system at $550{ }^{\circ} \mathrm{C}$. Next, a semi-Gaussian-shaped $\mathrm{KrF}$ excimer laser $(\lambda=248 \mathrm{~nm})$ at an energy density of $420 \mathrm{~mJ} / \mathrm{cm}^{2}$ was performed for the phase transformation from amorphous to polycrystalline silicon. The average grain size of the poly-Si measured using scanning electron microcscopy (SEM) is approximately $300 \mathrm{~nm}$, as shown in Fig. 1(b). Individual active regions were then patterned and defined. After the clean process, the samples were subjected to the $\mathrm{CF}_{4}$ plasma treatment, conducted in a plasma-enhanced chemical vapor deposition (PECVD) system at $350^{\circ} \mathrm{C}$ for $15 \mathrm{~s}$, under a pressure of 200 mtorr and a power of $10 \mathrm{~W}$, followed by a deposition of 100-nm-thick TEOS oxide by PECVD at $350{ }^{\circ} \mathrm{C}$. Then, a 200-nm-thick poly-Si was deposited by LPCVD at $620^{\circ} \mathrm{C}$ to serve as the gate electrode. A self-aligned phosphorous ion implantation was preformed at the dosage and energy of $5 \times 10^{15} \mathrm{~cm}^{-2}$ and $40 \mathrm{keV}$, respectively. The dopant activation was performed by excimer laser annealing (ELA), followed by a deposition of passivation layer and the definition of contact holes. Finally, a 500-nm-thick Al electrode was deposited and patterned. For comparison, the control TFTs were prepared without the $\mathrm{CF}_{4}$ plasma treatment process. No hydrogenation and thermal annealing process were performed after the $\mathrm{Al}$ formation.

\section{RESUlTS AND DiSCUSSION}

There was no significant surface contamination on the poly-Si samples after chemical cleaning detected using total reflection X-ray fluorescence (TRXRF). Also, the variation of the thickness of the poly-Si films before and after $\mathrm{CF}_{4}$ plasma treatment measured using ellipsometer was within 5\%. Therefore, the surface cleaning effect and the thinning effect of the 


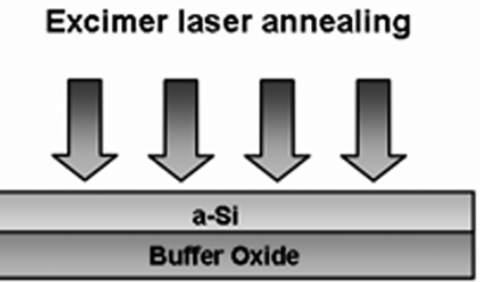

(a)

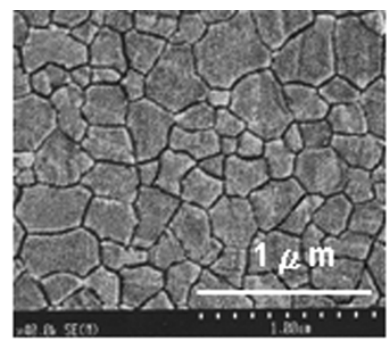

(b)

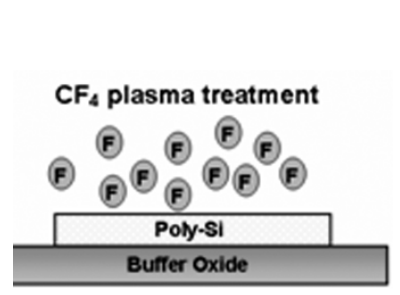

(c)

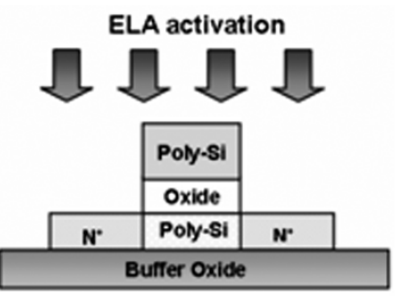

(d)

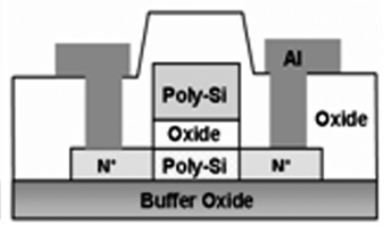

(e)

Fig. 1. Schematic diagram of the fabrication process of poly-Si TFT with $\mathrm{CF}_{4}$ plasma treatment. (a) Depositing amorphous silicon and ELA processing. (b) SEM image of ELA-processed poly-Si film after secco-etch. (c) Defining active region and $\mathrm{CF}_{4}$ plasma treatment. (d) S/D implantation and dopand activation by ELA. (e) Depositing passivation oxide, opening contact holes, depositing metal, and patterning metal.

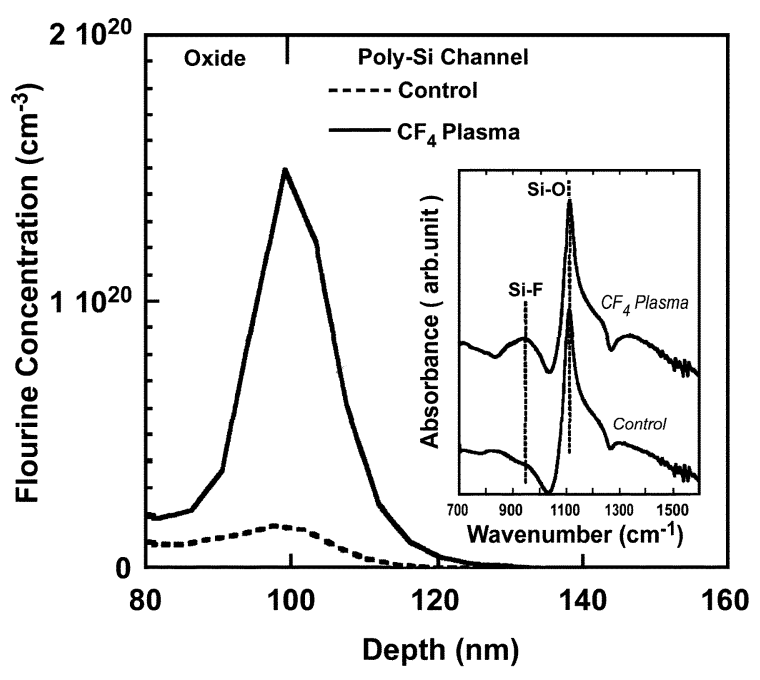

Fig. 2. SIMS profiles of conventional and $\mathrm{CF}_{4}$ plasma-treated poly-Si films. Inset shows the FTIR spectra of the control and the $\mathrm{CF}_{4}$ plasma-treated poly-Si films.

$\mathrm{CF}_{4}$ plasma treated poly-Si films were excluded in this experiment. Fig. 2 shows the SIMS profiles of the control and $\mathrm{CF}_{4}$ plasma-treated poly-Si films. Fluorine atoms are introduced into the $\mathrm{SiO}_{2} /$ poly-Si interface by the $\mathrm{CF}_{4}$ plasma treatment. These piled-up fluorine atoms can provide an effective trap states termination, because the channel is formed near the interface. The inset shows the Fourier transform infrared spectroscopy (FTIR) spectra of the control and $\mathrm{CF}_{4}$ plasma-treated poly-Si samples. The spectra exhibit absorption peaks corresponding to $\mathrm{Si}-\mathrm{F}$ bonds centered at round $940 \mathrm{~cm}^{-1}$ [9], [10]. The strong peak of $\mathrm{Si}-\mathrm{O}$ bond is related to the $\mathrm{SiO}_{2}$ substrate. Therefore, $\mathrm{Si}-\mathrm{F}$ bonds were formed in the poly-Si by employing the $\mathrm{CF}_{4}$ plasma treatment.

Fig. 3 shows the transfer characteristics and field-effect mobility versus the gate voltage for the devices. The measurements were performed at $V_{\mathrm{DS}}=0.5$ and $5 \mathrm{~V}$. The threshold voltage

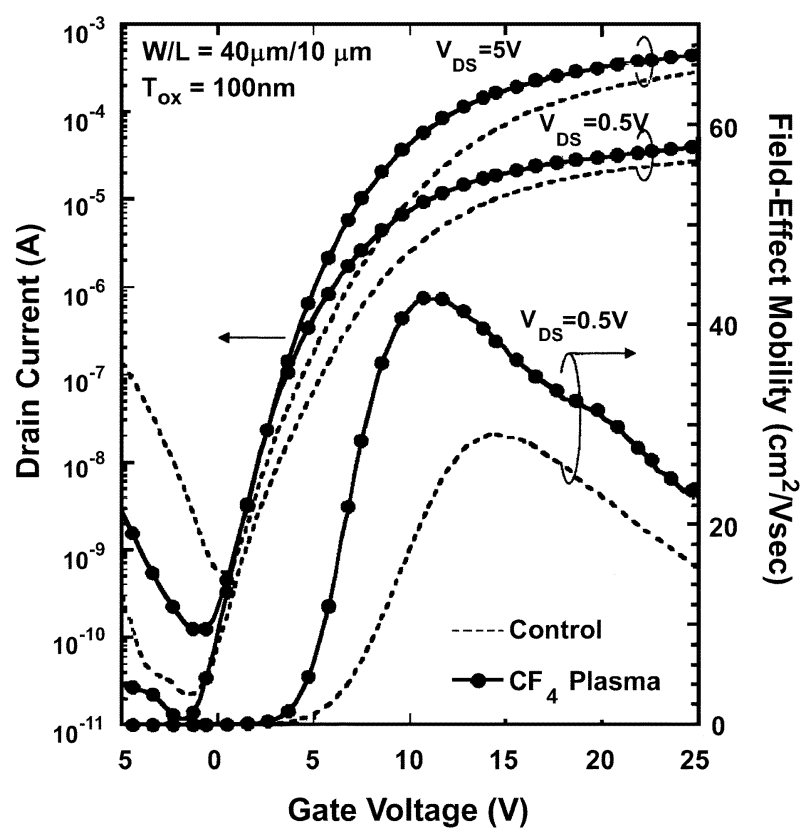

Fig. 3. Transfer characteristics and field-effect mobility versus gate voltage for the control and the $\mathrm{CF}_{4}$ plasma-treated poly-Si TFTs with $V_{\mathrm{DS}}=0.5$ and $5 \mathrm{~V}$.

$\left(V_{\mathrm{th}}\right)$ was defined as the gate voltage required to achieve a normalized drain current of $I_{D}=(W / L) \times 100 \mathrm{nA}$ at $V_{\mathrm{DS}}=5 \mathrm{~V}$. Accordingly, the $V_{\text {th }}$ and the subthreshold slope $(S S)$ of the $\mathrm{CF}_{4}$ plasma-treated TFT are $4.35 \mathrm{~V}$ and $1.28 \mathrm{~V} / \mathrm{dec}$., respectively, which are superior to $5.75 \mathrm{~V}$ and $1.92 \mathrm{~V} / \mathrm{dec}$. of the control TFT. Notably, the leakage current of the $\mathrm{CF}_{4}$ plasma treated TFT is more than one order in magnitude lower than that of the control TFT, especially under a large negative gate bias. The corresponding On/Off current ratios for the $\mathrm{CF}_{4}$ plasma-treated poly-Si TFT and the control TFT are $4.03 \times 10^{6}$ and $0.52 \times 10^{6}$ at $V_{\mathrm{DS}}=5 \mathrm{~V}$, respectively. The On/Off current ratio of the $\mathrm{CF}_{4}$ plasma-treated TFT is approximately eight times larger than that 


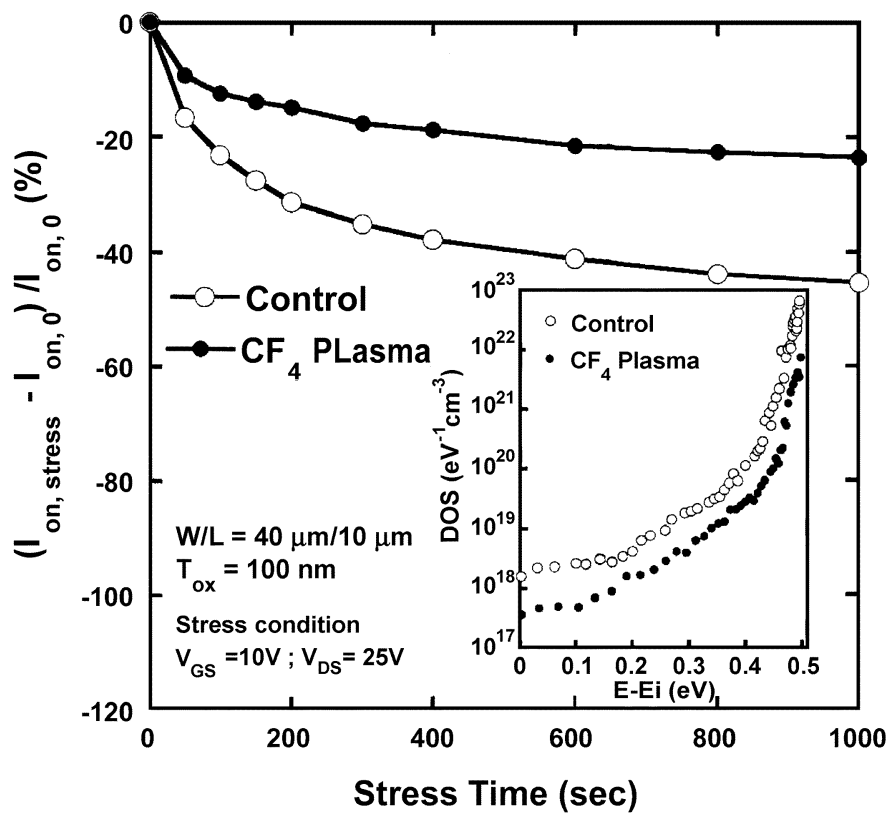

Fig. 4. On-current variations as a function of stress time under a hot carrier stress for the control and the $\mathrm{CF}_{4}$ plasma-treated poly-Si TFTs. Inset shows the density of states (DOS) in the energy bandgap of the control and the $\mathrm{CF}_{4}$ plasma-treated TFTs

of the control TFT. Additionally, the $\mathrm{CF}_{4}$ plasma-treated TFT has an about $50 \%$ enhancement in the maximum field-effect mobility. Note that great improvements on device characteristics were obtained even though the conditions of ELA were not optimized, which resulted in the rather poor field-effect mobility compared with other reports.

In order to verify the passivation effect of the $\mathrm{CF}_{4}$ plasma treatment, the effective trap states densities $\left(N_{\mathrm{t}}\right)$ near the $\mathrm{SiO}_{2} /$ poly-Si interface were calculated form the $S S$. By neglecting the depletion capacitance, the $N$ can be expressed as [11]

$$
\left.N_{t}=\left[\left(\frac{S S}{\ln 10}\right)\left(\frac{\mathrm{q}}{\mathrm{kT}}\right)-1\right)\right]\left(\frac{C_{\mathrm{ox}}}{\mathrm{q}}\right)
$$

where the $C_{\text {ox }}$ is the capacitance of the gate oxide. The $N_{t}$ of the control TFT and the $\mathrm{CF}_{4}$ plasma treated TFT are $6.72 \times 10^{12} \mathrm{~cm}^{-2}$ and $4.42 \times 10^{12} \mathrm{~cm}^{-2}$, respectively. The $N_{\mathrm{t}}$ values reflect both interface sates and grain boundary trap states near the $\mathrm{SiO}_{2}$ /poly-Si interface. Therefore, those states near the $\mathrm{SiO}_{2} /$ poly-Si interface can be effectively terminated using $\mathrm{CF}_{4}$ plasma treatment. Combined with the SIMS profiles, we believe that the passivation effect is due to the piled-up fluorine atoms.

As shown in the inset of Fig. 4, the density of states (DOS) in the energy bandgap of the TFTs were calculated using fieldeffect conductance method [12]. Both deep states and tail states are significantly reduced using the $\mathrm{CF}_{4}$ plasma treatment. We deduce that for the control TFT, there exists lots of dangling bonds and strain bonds near the $\mathrm{SiO}_{2} /$ poly-Si interface, resulting in high deep states and tail states [13]. On the contrary, for the $\mathrm{CF}_{4}$ plasma treated TFT, fluorine atoms were introduced into the $\mathrm{SiO}_{2} /$ poly-Si network to terminate the dangling bonds, release the strain bonds and form the $\mathrm{S}-\mathrm{F}$ bonds, improving the device characteristics [5].

Additionally, hot carrier stress was carried out at $V_{\mathrm{DS}}=25 \mathrm{~V}$ and $V_{\mathrm{GS}}=10 \mathrm{~V}$ for $1000 \mathrm{~s}$ to examine the device reliability. Fig. 4 shows the variations of the On-current $\left(I_{\mathrm{on}}\right)$ under hot carrier stress. The $I_{\text {on }}$ degradation rate of the control is almost twice that of the $\mathrm{CF}_{4}$ plasma treated TFT. It is attributed to the strong $\mathrm{Si}-\mathrm{F}$ bonds formed at the $\mathrm{SiO}_{2} /$ poly-Si interface. The $\mathrm{Si}-\mathrm{F}$ bonds are hard to be broken under hot carrier stress, leading to a great improvement in the device reliability.

\section{CONCLUSION}

Significant improvements on the device performance, including threshold voltage, subthreshold slope, leakage current, field-effect mobility, and On/Off current ratio have been demonstrated. This is due to the reduction of the interface states and grain boundary trap states near the $\mathrm{SiO}_{2} /$ poly-Si interface. The $\mathrm{CF}_{4}$ plasma treatment also improves the hot-carrier immunity due to the formation of $\mathrm{Si}-\mathrm{F}$ bonds. It is concluded that the $\mathrm{CF}_{4}$ plasma treatment is a simple, effective and process-compatible method to fabricate high-performance and high-reliability poly-Si TFTs.

\section{REFERENCES}

[1] T. Serikawa, S. Shirai, A. Okamoto, and S. Suyama, "Low-temperature fabrication of high-mobility poly-Si TFTs for large-area LCDs," IEEE Trans. Electron Devices, vol. 36, no. 9, pp. 1929-1933, Sep. 1989.

[2] I. W. Wu, W. B. Jackson, T. Y. Huang, A. G. Lewis, and A. C. Chiang, "Passivation kinetics of two types of defects in polysilicon TFT by plasma hydrogenation," IEEE Electron Devices Lett., vol. 12, no. 5, pp. 181-183, May 1991.

[3] M. Hack, A. G. Lewis, and I.-W. Wu, "Physical models for degradation effects in polysilicon thin-film transistors," IEEE Trans. Electron Devices, vol. 40, no. 5, pp. 890-897, May 1993.

[4] H. N. Chern, C. L. Lee, and T. F. Lei, "The effects of fluorine passivation on polysilicon thin film transistors," IEEE Trans. Electron Devices, vol. 41, no. 5, pp. 698-702, May 1994.

[5] S. Maegawa, T. Ipposhi, S. Maeda, H. Nishimura, T. Ichiki, M. Ashida, O. Tanina, Y. Inoue, T. Nishimura, and N. Tsubouchi, "Performance and reliability improvements in poly-Si TFTs by fluorine implantation into gate poly-Si," IEEE Trans. Electron Devices, vol. 42, no. 6, pp. 1106-1111, Jun. 1995.

[6] J. W. Park, B. T. Ahn, and K. Lee, "Effects of $F^{+}$implantation on the characteristics of poly-Si films and low-temperature n-ch poly-Si thin-film transistors," Jpn. J. Appl. Phys., vol. 34, pp. 1436-1441, Mar. 1995.

[7] C. H. Kim, J. H. Jeon, J. S. Yoo, K. C. Park, and M. K. Han, "Excimerlaser-induced in situ fluorine passivation effects on polycrystalline silicon thin film transistors," Jpn. J. Appl. Phys., vol. 38, pp. 2247-2250, Apr. 1999.

[8] C. H. Kim, S. H. Jung, J. S. Yoo, and M. K. Han, "Poly-Si TFT fabricated by laser-induced in situ fluorine passivation and laser doping," IEEE Electron Devices Lett., vol. 22, no. 8, pp. 396-398, Aug. 2001.

[9] Y. H. Kim, M. S. Hwang, and H. J. Kim, "Infrared spectroscopy study of low-dielectric-constant fluorine-incorporated and carbon-incorporated silicon oxide films," J. Appl. Phys., vol. 90, pp. 3367-3370, Oct. 2001.

[10] S. Lee and J.-W. Park, "Effect of fluorine on dielectric properties of SiOF films," J. Appl. Phys., vol. 80, pp. 5260-5263, Nov. 1996.

[11] C. A. Dimitriadis, P. A. Coxon, L. Dozsa, L. Papadimitriou, and N. Economou, "Performance of thin-film transistors on polysilicon films grown by low-pressure chemical vapor deposition at various pressures," IEEE Trans. Electron Devices, vol. 39, pp. 598-606, Mar. 1992.

[12] G. Fortunato and P. Migliorato, "Determination of gap state density in polycrystalline silicon by field-effect conductance," Appl. Phys. Lett., vol. 49, pp. 1025-1027, Oct. 1986.

[13] M. Cao, T. J. King, and K. C. Saraswat, "Determination of the densities of the gap states in hydrogenated polycrystalline $\mathrm{Si}$ and $\mathrm{Si}_{0.8} \mathrm{Ge}_{0.2}$ films," Appl. Phys. Lett., vol. 61, pp. 672-674, Aug. 1992. 